\title{
DM interpretations of heavy resonances and BSM-Higgs searches in ATLAS and CMS
}

\author{
Maria Savina ${ }^{a, 1, *}$ \\ a Joint Institute for Nuclear Research, \\ Dubna, Russia \\ E-mail: savina@cern.ch
}

For interpreting DM search results at the LHC the simplified mediator-based dark matter models are used, that allow to minimize the number of new particles and model parameters in consideration. In particular, only one spin- 0 or spin- 1 mediator and one DM particle $\chi$ assumed to be a fermion are introduced. Also s-channel processes are mainly considered in the simplest case.

Several searches are presented for DM signals within the simplified mediator-based models with an emphasis on fully visible signatures (heavy resonances) and MET signatures with good model sensitivity, especially to $2 \mathrm{HDM}+Z_{V}^{\prime}$ and $2 \mathrm{HDM}+a$ model parameters.

The Eighth Annual Conference on Large Hadron Collider Physics-LHCP2020

25-30 May, 2020

online

${ }^{1}$ On behalf of the ATLAS and CMS collaborations

* Speaker 


\section{Introduction: simplified dark matter models and signatures}

The search for dark matter at the LHC is one of the activities of the "Exotica" groups of the ATLAS and CMS Collaborations [1, 2]. The approach developed during the RUN1 and the RUN2 involves the use of simplified dark matter (DM) models and the description of the interaction processes between DM and the Standard Model (SM) particles by methods of effective field theory [3-5]. The effective approach makes it possible to operate with a limited number of new particles and to add only one mediator of interaction from the dark sector of matter to the ordinary one and one DM particle $\chi$ which is assumed throughout the paper to be a fermion ${ }^{1}$. In the simplest case, only s-channel processes are considered with the exchange of mediator and its decay into a pair of DM or SM particles. More complex scenarios also contain t-channel processes.

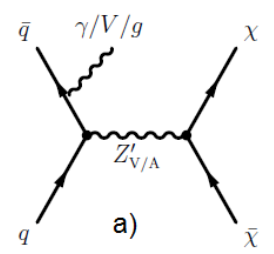

a)

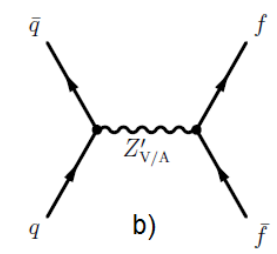

b)

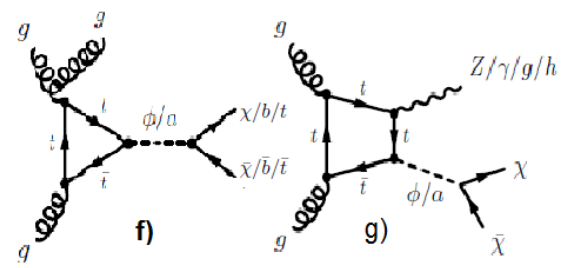

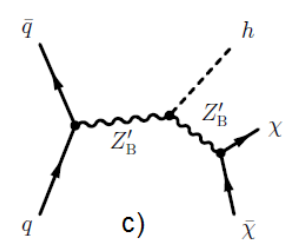

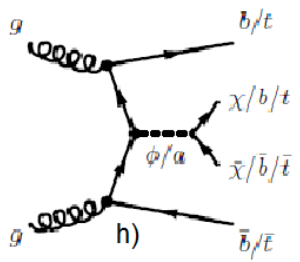

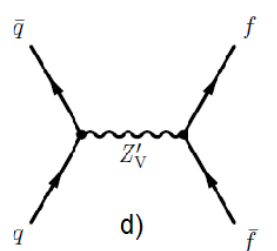

d)

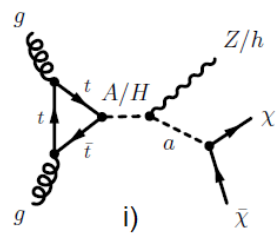

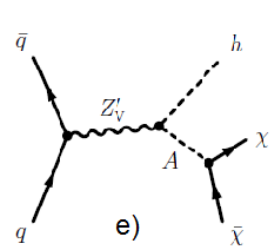

e)

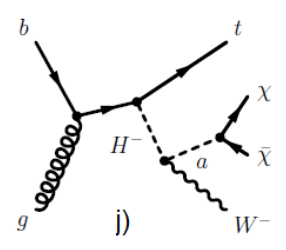

Figure 1: Basic Feynmann graphs for mediator-based simplified models considered. (Top panel) (Axial)vector mediator interaction with the SM matter for a) - b) the simplest s-channel model (V/VA), c) the VBC model, d) - e) the $2 \mathrm{HDM}+Z_{V}^{\prime}$ model. (Bottom panel) (Pseudo)scalar mediator interaction with the SM matter for $\mathrm{f}$ ) - $\mathrm{h}$ ) the simplest s-channel model (S/PS), i) - j) the $2 \mathrm{HDM}+a$ models (more details see in [6]). The diagrams of $\mathrm{f}$ ) $-\mathrm{h}$ ) are common for S/PS and $2 \mathrm{HDM}+a$ models.

Mediator can have spin 0 or 1 and be (pseudo)scalar or (axial)vector, respectively. The list of simplified dark matter models used during RUN2 with a detailed description can be found in [6] and references therein. The associated model acronyms and mediator symbols used throughout correspond to

- $\operatorname{V/AV}\left(Z_{V}^{\prime} / Z_{A}^{\prime}\right)$ - spin-1 vector or axial-vector mediator of the simplest s-channel DM model;

- S/PS $(\phi / a)$ - spin-0 scalar or pseudoscalar mediator of the simplest s-channel DM model;

- $\operatorname{VBC}\left(Z_{b}^{\prime}\right)$ - spin-1 vector baryon-number-charged mediator;

\footnotetext{
${ }^{1}$ In general it is not mandatory, the interaction with a dark sector can go through more than one "portal", i.e. not due to a single mediator, and a DM particle does not have to be only a fermion, but can have any spin (see for example [7])
} 
- $2 \mathrm{HDM}+a-$ spin-0 pseudoscalar mediator of the simplified DM model with an extended Higgs sector;

- $2 \mathrm{HDM}+Z_{V}^{\prime}-$ spin-1 vector mediator of the simplified DM model with an extended Higgs sector.

The 2HDM here is well-known two-doublet Higgs model, contains five states in the Higgs sector (CP-even $h, H, \mathrm{CP}$-add $A$, charged $H^{ \pm}$), DM studies are performed in the aligment limit when the lighter state $h$ has the properties of the observed Higgs boson $h_{125}$. 2HDM and especially its next extension $2 \mathrm{HDM}+\mathrm{S}$ containing one more extra Higgs scalar give a popular theoretical base for so-called "the Higgs portal" with $2 \mathrm{HDM}+a$ and $2 \mathrm{HDM}+Z_{V}^{\prime}$ models in which the interactions between the dark and ordinary sectors are transferred using Higgs states.

Table 1: Summary of searches for mediator-based simplified models considered in the paper, along with the final-state signatures ( $2^{\text {nd }}$ column) specific or common to different classes of models ( $3^{\text {rd }}$ column, see model details in [6]). The $4^{\text {th }}$ column gives the references to the corresponding ATLAS and CMS results.

\begin{tabular}{|c|c|c|c|}
\hline & Signatures & Models & References \\
\hline \multirow{4}{*}{ Visible } & dijet (dilepton) resonance, dijet $\chi$ & $V / A V$ & [8-14] \\
\hline & $t \bar{t}(b \bar{b})$ resonance & $\begin{array}{c}\mathrm{V} / \mathrm{AV}, 2 \mathrm{HDM}+Z_{V}^{\prime}, \\
\mathrm{S} / \mathrm{PS}, 2 \mathrm{HDM}+a\end{array}$ & [18-20] \\
\hline & $t \bar{t} t \bar{t}$ & $2 \mathrm{HDM}+a$ & [15] \\
\hline & $\begin{array}{c}X \rightarrow h h(4 W / 2 W 2 \gamma / \\
2 W b \bar{b} / b \bar{b} \tau \tau(2 \gamma) / 2 b \bar{b} \\
X \rightarrow Z Z / W^{+} W^{-}\end{array}$ & $\mathrm{S} / \mathrm{PS}, 2 \mathrm{HDM}+a$ & {$[21,22]$} \\
\hline \multirow{5}{*}{ MET } & $t \bar{t}(b \bar{b})+E_{T}^{m i s}$ & $\mathrm{~S} / \mathrm{PS}, 2 \mathrm{HDM}+a$ & {$[16]$} \\
\hline & $h(b \bar{b} / \gamma \gamma / \tau \tau)+E_{T}^{m i s}$ & $\mathrm{VBC}, 2 \mathrm{HDM}+Z_{V}^{\prime}$ & {$[23,25-27]$} \\
\hline & $h(b \bar{b} / \gamma \gamma) / Z(q \bar{q} / l l)+E_{T}^{m i s}$ & $\mathrm{~S} / \mathrm{PS}, 2 \mathrm{HDM}+a$ & [27] \\
\hline & $h\left(W^{+} W^{-} / Z Z\right)+E_{T}^{m i s}$ & $\mathrm{VBC}, 2 \mathrm{HDM}+Z_{V}^{\prime}$ & [24] \\
\hline & $s(b \bar{b})+E_{T}^{m i s}$ & Dark Higgs & {$[30]$} \\
\hline \multirow{2}{*}{ (Semi)invisible } & $h \rightarrow$ inv & $\mathrm{S} / \mathrm{PS}, 2 \mathrm{HDM}+a$ & {$[28,29]$} \\
\hline & $h \rightarrow \gamma \gamma_{D}$ & Higgs portal & {$[31]$} \\
\hline
\end{tabular}

Basic Feynmann graphs for mediator-based simplified models considered represented on Figure 1. Extra lines with new Higgs states in $2 \mathrm{HDM}+a$ and $2 \mathrm{HDM}+Z_{V}^{\prime}$ give processes similar to other models, (c),(g) and (e),(i) respectively, or represent quite specific options inherent only to these models (j). As it follows from the graphs in the simplest case effective description of interaction processes depend only on four model parameters, i.e. mediator and DM particle masses $m_{m e d}$ and $m_{D M}\left(m_{\chi}\right)$ and coupling to quarks $g_{q}$ (universal on flavours, because of minimal flavour violation requirement) and to DM fermions $g_{D M}$ for a leptophobic mediator (otherwise there is also coupling to leptons $g_{l}$ ). Taking into account the strong constraints obtained from the Drell-Yan study for vector mediator (the graphs type (b) for $Z_{V}^{\prime}$ and dilepton final states) acceptable values of $g_{l}$ should be sufficiently small. For more complicated DM models several more are added to these four 
parameters. For the 2HDM-based DM models extra Higgs masses $m_{A}, m_{H, H^{ \pm}}, m_{S}$ appear (often assumed $m_{A}=m_{H}$ ), along with a ratio of vacuum expectation values for two Higgs doublets $\tan \beta$. In $2 \mathrm{HDM}+a$ a mixing angle $\sin \theta$ between pseudoscalar mediator $a$ and $A$ is also present. The DM search is carried out in the space of two selected parameters with the remaining fixed, for example, in $\left(m_{m e d}, m_{D M}\right),\left(m_{m e d}, \sin \theta\right),\left(m_{m e d}, m_{A, H}\right)$ plane etc.

From an experimental point of view, all possible final states are divided into completely visible signatures (all registered final particles), signatures with missing transverse momentum from mediator or a SM boson decay into a pair of DM particles $\chi \bar{\chi}$, and completely invisible ones which are used for particle decays with known properties for the possibility of restoring the energy-momentum balance at the vertex (invisible Higgs decay). A list of the models used, along with signatures and links to the relevant analyses, is given in the Table 1.

The selected signatures can be universal for many models or model specific. Ideally, results of the DM search should contain combined data of complementary studies of visible, MET and invisible (when applicable) final signatures. At the moment it is implemented for V/AV and S/PS mediators and partially for 2HDM-based models. Below are a few examples of the recent studies in different channels for for these cases.

\section{V/AV models in dijet}

Fully visible dijet channel (graph (b) on Figure1, diquark final state), in a sense, a golden classic for heavy enough narrow resonances. The cross section of such a process depends only on two parameters, i.e. a mediator mass $m_{m e d}$ and an universal coupling to quarks for leptophobic case of V/AV models (for "leptophilic" case dependence on a $g_{l}$ should be added). It allows to obtain limits on the models in $\left(m_{m e d}, g_{q}^{\prime}\right)$ plane $[8,9]$.

Problematic region of lower invariant masses requires some special reconstruction techniques. This is the addition of a third jet (from initial state radiation) to increase the experimental sensitivity to softer jets masked by a large QCD background, as well as special trigger and on-line recording used [10]. All of this makes it possible to reconstruct the jets and to push down the experimental sensitivity to dijet invariant mass as small as about of $10 \mathrm{GeV}$. Another problem arises when spin-1 mediator becomes too wide and the narrow width approximation cannot be applied. Then another signature can help which is commonly used for non-resonant deviations from the SM background, i.e. the angular distributions of dijets [11,12]. Using the signature allows to move into coupling values as large as of the order of unity. As a result, constraints are obtained on the V/AV model parameters in a very wide range of dijet invariant masses from $10 \mathrm{GeV}$ up to $4.5 \mathrm{TeV}$.

Figure 2 contains the general dijet plot for leptophobic vector mediator which shows the CMS results obtained between 2015 and 2018, corresponding to an integrated luminosity of $139 \mathrm{fb}^{-1}$ with the recent ATLAS data added, and with previous experiment limits and precision measurements of resonance width (for $\Upsilon$ and $\mathrm{Z}$ boson). The areas above the curves are considered to be excluded. The same for the ATLAS can be found in [13].

Dijet channel limits can be combined with data obtained from the MET signatures with participation of dark matter (graph (a) on Figure 1). Such combined results for leptophobic axialvector mediator in AV model are shown in Fig 3 (the same for leptophilic mediator and V model see in $[13,14])$. 


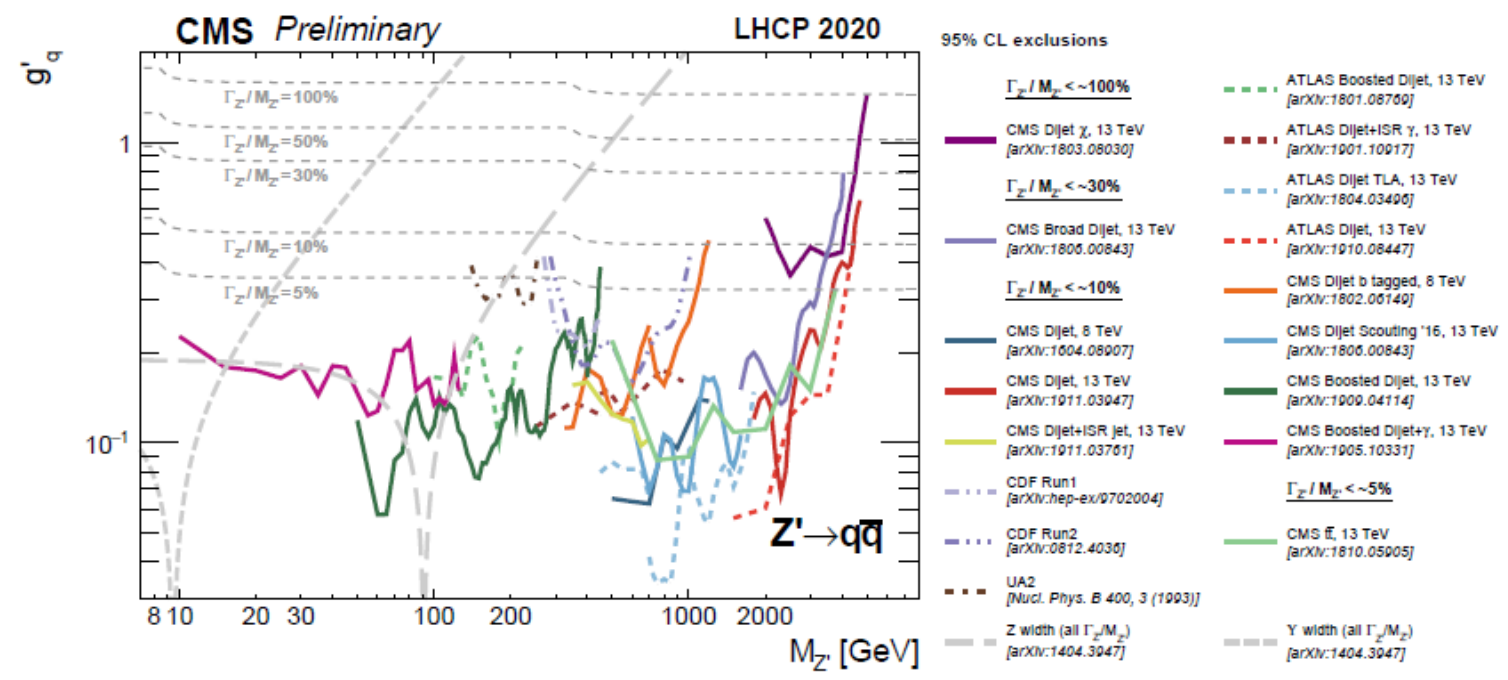

Figure 2: Limits on the universal coupling $g_{q}^{\prime}$ between a leptophobic $Z^{\prime}$ boson and quarks from various dijet analyses from CMS, ATLAS, CDF, and UA2. The limits are shown in solid lines, with the excluded area above the lines. The hashed areas show the direction of the excluded area from the observed limits. The grey dashed lines show the $g_{q}^{\prime}$ values at fixed values of $\Gamma_{Z^{\prime}} / M_{Z^{\prime}}$. Most of the analyses, with the exception of Dijet $\chi$ and Broad Dijet, assume that the intrinsic width is negligible compared to the experimental resolution, and hence are valid for $\Gamma_{Z^{\prime}} / M_{Z^{\prime}} \leq 10 \%$. The $t \bar{t}$ resonance analysis is valid for $\Gamma_{Z^{\prime}} / M_{Z^{\prime}} \leq 5 \%$, the Broad Dijet analysis is valid for $\Gamma_{Z^{\prime}} / M_{Z^{\prime}} \leq 30 \%$, and the Dijet $\chi$ analysis is valid for $\Gamma_{Z^{\prime}} / M_{Z^{\prime}} \leq 100 \%$. Also shown are indirect constraints on $g_{q}^{\prime}$ from the $\Upsilon$ and $Z$ boson widths, which are valid for all values of $\Gamma_{Z^{\prime}} / M_{Z^{\prime}}[14]$.

A comparison of the inferred limits with the constraints from experiments on DM direct detection in terms of spin-independent or spin-dependent scattering cross-section on nucleons (WIMP-proton and WIMP-neutron processes) also can be done for the leptophobic and leptophilic $Z^{\prime}$-based simplified model [13, 14].

\section{Models with (pseudo)scalar mediator in $t \bar{t}(b \bar{b}), t \bar{t} t \bar{t}$, and $t \bar{t}(b \bar{b})+E_{T}^{m i s}$ channels}

The graph (h) on Figure 1 actually gives two complementary channels, i.e. fully visible fourquark final state $t \bar{t} t \bar{t}$ and MET signature $t \bar{t}(b \bar{b})+E_{T}^{m i s}$. For the DM models with (pseudo)scalar mediator both of these channels provide the strongest limits on the model parameter space. Figure 4 demonstrates the CMS results for S/PS model interpretation in the four-top-quark channel. The data collected during the 2016 - 2018 period, corresponds to an integrated luminosity of $137 \mathrm{fb}^{-1}$ at a center-of-mass energy of $13 \mathrm{TeV}$ [15].

These results can be supplemented with the S/PS model limits on a mediator mass $m_{\phi, a}$ obtained by ATLAS in the $t \bar{t}+E_{T}^{m i s}$ channel [16] and by CMS in the combined channel $t \bar{t} / t W+E_{T}^{m i s}$ for S/PS models [17]. The ATLAS results also can be interpreted in terms of $\mathrm{SCC}_{b(t)}$ models with scalar colour-charged mediator (for $\mathrm{SCC}_{b(t)}$ models see [6]).

The fully visible four-quark final state $b \bar{b} b \bar{b}$ with boosted $b \bar{b}$ pairs is used as one of the possible signatures to set constraints on the parameter space of a special case of the $2 \mathrm{HDM}+\mathrm{S}$ model (type II, or NMSSM, see, for example, [7]) that can have DM interpretation [18, 19]. In this case, the 

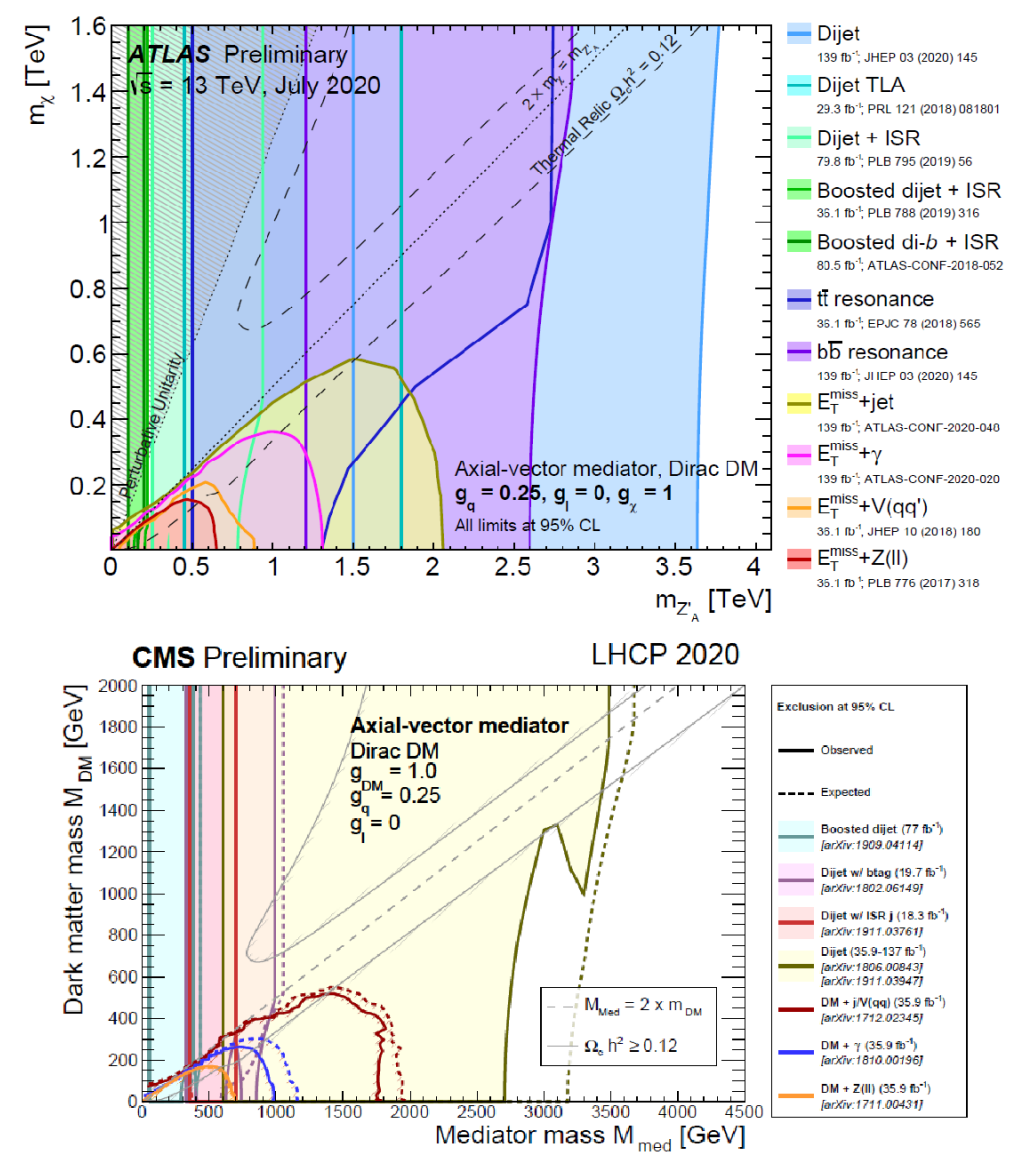

Figure 3: $95 \% \mathrm{CL}$ observed and expected exclusion regions in $m_{M e d}, m_{D M}$ plane for dijet searches and different MET based DM searches from ATLAS (top) [13]) and CMS (bottom) [14] for leptophobic axialvector mediator simplified models. Values of $g_{q}=0.25$ and $g_{D M}\left(g_{\chi}\right)=1.0$ are chosen following the recommendation of the LHC DM working group $[4,5]$.

existence of a light pseudoscalar $a$ with a mass less than half the Higgs $h_{125}$ mass is assumed and decay chains $h \rightarrow a a \rightarrow b \bar{b} b \bar{b} / b \bar{b} \mu^{+} \mu^{-} / b \bar{b} \tau^{+} \tau^{-}$etc. are considered.

The universal signature with heavy flavour resonance $t \bar{t}(b \bar{b})$ which is shown in the graph (f) on Figure 1 basically can be used both for V/AV and $2 \mathrm{HDM}+Z_{V}^{\prime}$ models and S/PS and 2HDM $+a$ (see Table 1 for signatures and model sensitivity). For V/AV these results go into the combined plot shown in the previous paragraph. For S/PS models DM interpretation can be done as a $\left(m_{\phi(a)}, g_{q \phi(q a)}\right)$ plot using the results on boosted $b \bar{b}$ pair production from light (pseudo)scalar mediator decay [20].

For completeness of the picture on the visible channels, one can also mention the limits obtained for scalar mediator of S model from the DM interpretation of a new heavy scalar decay into two SM bosons $X \rightarrow h_{125} h_{125} / Z Z\left(W^{+} W^{-}\right)[21,22]$. 

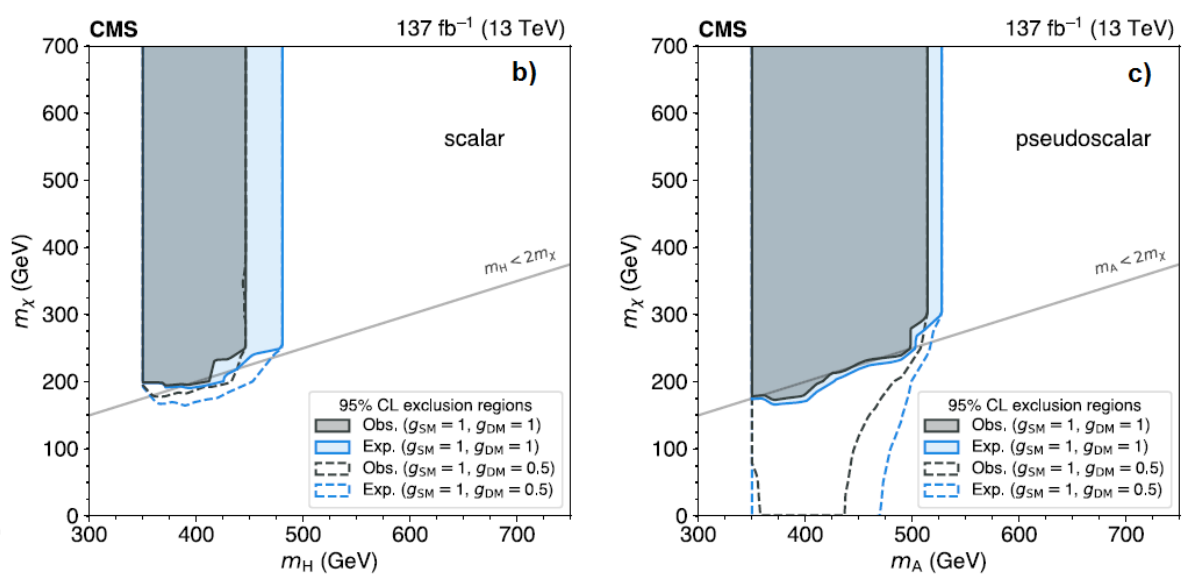

Figure 4: (a) Exclusion regions at 95\% CL in the plane of $m_{\chi}$ vs. $m_{H}$ (b) or $m_{A}$ (c). The outer lighter and inner darker solid curves show the expected and observed limits, respectively, assuming $g_{S M}=g_{D M}=1$. The excluded regions, shaded, are above the limit curves. The dashed lines show the limits assuming a weaker coupling between $H / A$ and $\chi, g_{D M}=0.5$ [15].

\section{4. $2 \mathrm{HDM}+Z_{V}^{\prime}$ and $2 \mathrm{HDM}+a$ models in mono $h_{125}+\mathrm{MET}$ channel}

Following Table 1 one can conclude that mono $h+$ MET signature is sensitive to a wide class of models, including VBC, $2 \mathrm{HDM}+Z_{V}^{\prime}$, S/PS and 2HDM $+a$ (graphs (c), (e), (g), and (i) on Figure 1 , respectively). Possible final states in the Higgs decay can also differ, i.e. $h \rightarrow b \bar{b}$ (boosted), $h \rightarrow \gamma \gamma / \tau^{+} \tau^{-} / W^{+} W^{-} / Z Z$, and its combinations. The additional graphs is obtained by replacing the Higgs line with the SM $Z$ vector boson line, so one more signature, i.e. $Z+$ MET can be considered. Figure 5 contains the limit plots for vector mediator in the $\left(m_{Z^{\prime}}, m_{A}\right)$ plane for the $2 \mathrm{HDM}+Z_{V}^{\prime}$ model.

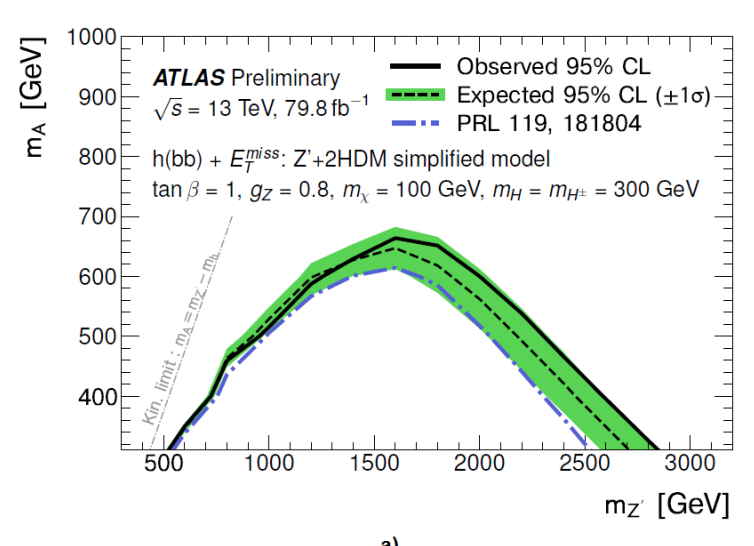

a)

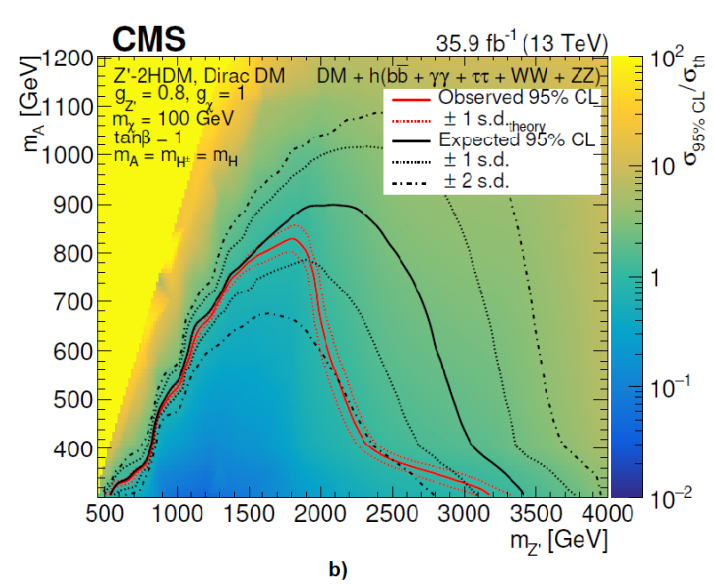

b)

Figure 5: Exclusion contours for the $2 \mathrm{HDM}+Z_{V}^{\prime}$ scenario in the $\left(m_{Z^{\prime}}, m_{A}\right)$ plane for $\tan \beta=1, g_{Z^{\prime}}=$ 0.8 , and $m_{\chi}=100 \mathrm{GeV}$ for (a) ATLAS in the $h(b \bar{b})+$ MET channel [23] and (b) CMS in the combined $h \rightarrow b \bar{b} / 2 \gamma / 2 \tau / Z Z / W W+$ MET channel [24].

The same results can be reinterpreted for vector mediator of VBC model in terms of mass limit plots and the upper limits on the DM-nucleon spin-independent scattering cross-section $\sigma^{S I}$, as 
a function of $m_{\chi}$ [23, 24]. In addition one can look also at the alalyses [25] for the light vector mediator with a mass of 100 to $200 \mathrm{GeV}$, [26] for the results in $h \rightarrow \gamma \gamma$ channel, and [27] for VBC, $2 \mathrm{HDM}+Z_{V}^{\prime}$, and scalar S model limit plots.

Figure 6 demonstrates the results of combined analysis for pseudoscalar mediator of the $2 \mathrm{HDM}+a$ model performed in [6] by ATLAS on the data of half of the RUN2. The combined limits also include data on invisible Higgs decay. The very recent ATLAS and CMS results on the invisible mode can be found in [28] and [29]), respectively.

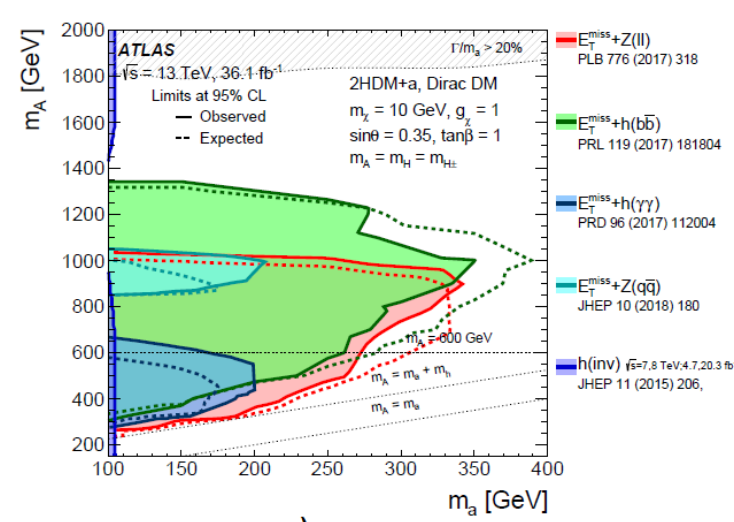

a)

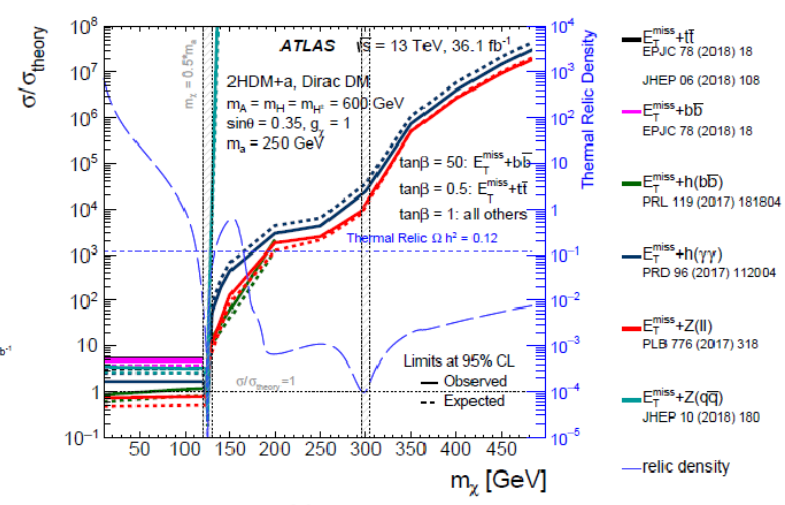

b)

Figure 6: (a) Regions in the $\left(m_{a}, m_{A}\right)$ plane excluded by data at $95 \% \mathrm{CL}$ by $X+E_{T}^{m i s}$ and $t \bar{t} t \bar{t}$ analyses, following the parameter choices of scenarios 1 and 2 of the $2 \mathrm{HDM}+\mathrm{a}$ model. The dashed grey region at the top indicates the region where the width of any of the Higgs bosons exceeds $20 \%$ of its mass. (b) Observed exclusion limits for the 2HDM+a model as a function of $m_{\chi}$, following the parameter choices of scenario 4 . The limits are calculated at 95\% CL and are expressed in terms of the ratio of the excluded cross-section to the nominal cross-section of the model. The relic density for each $m_{\chi}$ assumption is superimposed in the plot (long-dashed line) and described by the right vertical axis. For scenarios and parameter choice see [6].

\section{Walking into the dark sector: (semi)invisible Higgs decay, Dark Higgs model}

At last, mention can be made of the ATLAS analyses reinterpretation in the framework of the so-called dark Higgs model [30]. The new scalar, denoted as $s$, mixes weakly with the standard Higgs and gives mass to a new heavy vector boson $Z^{\prime}$. The interaction with the SM matter is carried out in two ways through the Higgs sector between scalars and through the gauge sector between a new vector boson and quarks. The signature with mono Higgs and missing $E_{T}$ is very similar to that for the baryonic $Z_{b}^{\prime}$ of the VBC model, only instead of the standard Higgs boson line on the graphs (c) on the Figure 1 now there is a new Higgs state $s$ from the dark sector, as on Figure 7 on the left.

Branchings for different channels are given in [30]. In small mass region decay into a $b \bar{b}$ pair dominates. Figure 7 on the right illustrates the upper limit on the cross section and the excluding plot for a dark Higgs mass depending on vector mediator mass. The area to the left of the curve is closed by the experiment.

Other possible examples of searches for dark sector particles are more related to "Longlived Particles" (LL) group activity of the ATLAS and CMS collaborations. This includes, in particular, 

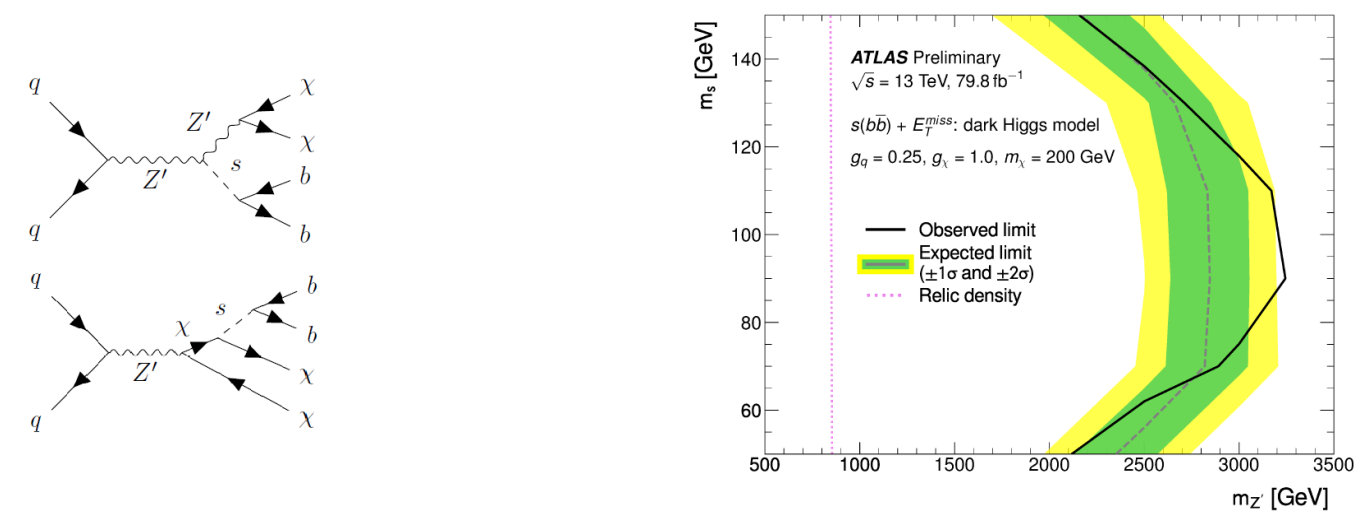

Figure 7: Feynmann graphs (left) for the dark Higgs model with lines corresponded to $Z^{\prime}$ and $s$ propogation in s-channel processes. Exclusion contour for the model (right) with parameters $m_{\chi}=200 \mathrm{GeV}, g_{q}=0.25$, $g_{\chi}=1.0$ and different values of $m_{Z^{\prime}}$ and $m_{s}$. The solid (dashed) line shows the observed (expected) limit, while the green and yellow bands indicate the \pm 1 and \pm 2 uncertainty on the expected limit, respectively. The pink dotted curve corresponds to the set of points for which the expected relic density is consistent with the PLANCK measurement (i.e. $\Omega h^{2}=0.114$ ), as computed with MadDM. The region on the right of the curve corresponds to a predicted relic abundance which is higher than these measurements [30].

the one mentioned in Table 1 semi-invisivle Higgs decay into a pair of ordinary photon and "dark" photon $h_{125} \rightarrow \gamma \gamma_{D}$ [31].

\section{Conclusions}

We have presented several searches for DM signals within the simplified mediator-based models with an emphasis on fully visible signatures (heavy resonances) and MET signatures with good model sensitivity, especially to $2 \mathrm{HDM}+Z_{V}^{\prime}$ and $2 \mathrm{HDM}+a$ model parameters. In particular, dijet channel, combinations of a pair of heavy flavours and MET and the signature with two pairs of heavy flavours, as well as the mono $h+$ MET signature that is very useful for a wide range of models. For all cases, limit plots for DM particle and mediator were shown. Links to some additional related studies and channels were also provided.

Further prospects for DM search at the LHC are associated with the development of models and transition to the study of t-channel processes. It can be done, in particular, within the framework of a new generation of spin- 0 mediator models based on $2 \mathrm{HDM}+a$ approach as given in [32].

\section{References}

[1] ATLAS Collab., URL: https://twiki.cern.ch/twiki/bin/view/AtlasPublic/ExoticsPublicResults.

[2] CMS Collab., URL: https://twiki.cern.ch/twiki/bin/view/CMSPublic/PhysicsResultsEXO.

[3] Dark Matter Benchmark Models for Early LHC Run-2 Searches: Report of the ATLAS/CMS Dark Matter Forum, Phys. Dark Univ. 26 (2019) 100371. 
[4] Recommendations on presenting LHC searches for missing transverse energy signals using simplified s-channel models of dark matter, CERN-LPCC-2016-001 , arXiv: 1603.04156 [hep-ex].

[5] Recommendations of the LHC Dark Matter Working Group: Comparing LHC searches for heavy mediators of dark matter production in visible and invisible decay channels, CERNLPCC-2017-01, arXiv: 1703.05703 [hep-ex].

[6] ATLAS Collab., Constraints on mediator-based dark matter and scalar dark energy models using $\sqrt{s}=13 \mathrm{TeV}$ pp collision data collected by the ATLAS detector, JHEP 05 (2019) 142.

[7] G. Arcadi, A. Djouadi, and M. Raidal, Dark Matter through the Higgs portal, LAPTH-010/19, arXiv:1903.03616 [hep-ph].

[8] ATLAS Collab., Search for new resonances in mass distributions of jet pairs using $139 \mathrm{fb}^{-1}$ of pp collisions at $\sqrt{s}=13 \mathrm{TeV}$ with the ATLAS detector, JHEP 03 (2020) 145.

[9] CMS Collab., Search for high mass dijet resonances with a new background prediction method in proton-proton collisions at $\sqrt{s}=13 \mathrm{TeV}$, JHEP 05 (2020) 033 .

[10] CMS Collab., Search for Low-Mass Quark-Antiquark Resonances Produced in Association with a Photon at $\sqrt{s}=13$ TeV, Phys. Rev. Lett. 123 (2019) 231803.

[11] ATLAS Collab., Search for new phenomena in dijet events using $37 \mathrm{fb}^{-1}$ of pp collision data collected at $\sqrt{s}=13$ TeV with the ATLAS detector, Phys. Rev. D 96 (2017) 052004.

[12] CMS Collab., Search for new physics in dijet angular distributions using protonproton collisions at $\sqrt{s}=13 \mathrm{TeV}$ and constraints on dark matter and other models, Eur. Phys. J. C 78 (2018) 789.

[13] ATLAS Collab., Dark matter summary plots for s-channel mediators, ATL-PHYS-PUB-2020021, URL: http://cdsweb.cern.ch/record/2725266.

[14] CMS Collab., URL: https://twiki.cern.ch/twiki/pub/CMSPublic/SummaryPlotsEXO13TeV.

[15] CMS Collab., Search for production of four top quarks in final states with same-sign or multiple leptons in proton-proton collisions at $\sqrt{s}=13 \mathrm{TeV}$, Eur. Phys. J. C 80 (2020) 75.

[16] ATLAS Collab., Search for new phenomena with top quark pairs in final states with one lepton, jets, and missing transverse momentum in pp collisions at $\sqrt{s}=13 \mathrm{TeV}$ with the ATLAS detector, ATLAS-CONF-2020-003, https://cds.cern.ch/record/2711489.

[17] CMS Collab., Search for dark matter produced in association with a single top quark or a top quark pair in proton-proton collisions at $\sqrt{s}=13 \mathrm{TeV}$, JHEP 03 (2019) 141.

[18] ATLAS Collab., Exotic Higgs Decay Summary Plots, ATL-PHYS-PUB-2018-045, URL: http://cdsweb.cern.ch/record/2650740. 
[19] CMS Collab., Summary of 2HDM+Singlet searches at $\sqrt{s}=13 \mathrm{TeV}$, URL: https://twiki.cern.ch/twiki/pub/CMSPublic/SummaryResultsHIG.

[20] CMS Collab., Search for low-mass resonances decaying into bottom quark-antiquark pairs in proton-proton collisions at $\sqrt{s}=13 \mathrm{TeV}$, Phys. Rev. D 99 (2019), 012005.

[21] ATLAS Collab., Combination of searches for Higgs boson pairs in pp collisions at $\sqrt{s}=13$ TeV with the ATLAS detector, Phys. Lett. B 800 (2020) 135103.

[22] CMS Collab., Search for a heavy Higgs boson decaying to a pair of W bosons in proton-proton collisions at $\sqrt{s}=13 \mathrm{TeV}$, JHEP 03 (2020) 034, arXiv: 1912.01594 [hep-ex]; Combination of Searches for Higgs Boson Pair Production in Proton-Proton Collisions at $\sqrt{s}=13 \mathrm{TeV}$, Phys. Rev. Lett. 122 (2019) 121803.

[23] ATLAS Collab., Search for Dark Matter Produced in Association with a Higgs Boson decaying to $b \bar{b}$ at $\sqrt{s}=13 \mathrm{TeV}$ with the ATLAS Detector using $79.8 \mathrm{fb}^{-1}$ of proton-proton collision data, ATLAS-CONF-2018-039, URL: https://cds.cern.ch/record/2632344.

[24] CMS Collab., Search for dark matter particles produced in association with a Higgs boson in proton-proton collisions at $\sqrt{s}=13 \mathrm{TeV}$, JHEP 03 (2020) 25 .

[25] ATLAS Collab., Search for boosted resonances decaying to two b-quarks and produced in association with a jet at $\sqrt{s}=13 \mathrm{TeV}$ with the ATLAS detector, ATLAS-CONF-2018-052, URL: https://cds.cern.ch/record/2649081.

[26] ATLAS Collab., Search for dark matter in association with a Higgs boson decaying to two photons at $\sqrt{s}=13 \mathrm{TeV}$ with the ATLAS detector, Phys. Rev. D 96 (2017) 112004.

[27] CMS Collab., Search for dark matter produced in association with a Higgs boson decaying to a pair of bottom quarks in proton-proton collisions at $\sqrt{s}=13 \mathrm{TeV}$, Eur. Phys. J. C 79 (2019) 280.

[28] ATLAS Collab., Search for invisible Higgs boson decays with vector boson fusion signatures with the ATLAS detector using an integrated luminosity of $139 \mathrm{fb}^{-1}$, ATLAS-CONF-2020008, http://cdsweb.cern.ch/record/2715447.

[29] CMS Collab., Search for invisible decays of a Higgs boson produced through vector boson fusion in proton-proton collisions at $\sqrt{s}=13 \mathrm{TeV}$, Phys. Lett. B 793 (2019) 520; Search for new physics in events with a leptonically decaying $Z$ boson and a large transverse momentum imbalance in proton-proton collisions at $\sqrt{s}=13 \mathrm{TeV}$, Eur. Phys. J. C 78 (2018) 291.

[30] ATLAS Collab., RECAST framework reinterpretation of an ATLAS Dark Matter Search constraining a model of a dark Higgs boson decaying to two b-quarks, ATL-PHYS-PUB-2019032, URL: https://cds.cern.ch/record/2686290.

[31] CMS Collab., Search for dark photons in decays of Higgs bosons produced in association with $Z$ bosons in proton-proton collisions at $\sqrt{s}=13 \mathrm{TeV}$, JHEP 10 (2019) 139. 
[32] LHC Dark Matter Working Group: Next generation spin-0 dark matter models, arXiv: 1810.09420 [hep-ex]. 